\title{
Einheitliche Spritzenaufkleber - ein kleiner Schritt zu mehr Sicherheit
}

\author{
Thomas Prien
}

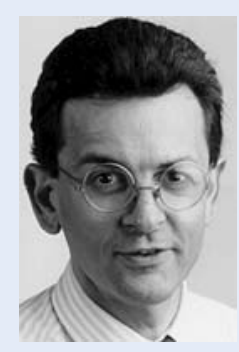

Thomas Prien

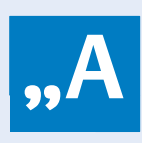

us kritischen Ereignissen lernen" und dadurch noch sicherer werden: Die Luftfahrt hat es vorgemacht. Die deutsche Medizin ist dabei, diesem Beispiel zu folgen. So genannte Critical Incident Reporting Systeme sind vielerorts eingeführt worden, die Bundesärztekammer bündelt sie jetzt in einem bundeseinheitlichen, fachübergreifenden Programm. Ein bedeutender Vorreiter dieser Berichtssysteme ist das PaSOS-Programm der Deutschen Gesellschaft für Anästhesiologie und Intensivmedizin.

Dessen Inaugurator, Herr Dr. Marcus Rall aus Tübingen, berichtet in dieser Ausgabe der Intensivmedizin up2date, was man aus den anonym dargestellten Fällen lernen kann. Medikationsfehler und Medikamentenverwechslungen sind auch immer wieder darunter. Das ganze Ausmaß des Problems offenbarte eine prospektive europäische Studie, die vor einem Jahr im British Medical Journal und im Deutschen Ärzteblatt veröffentlicht wurde. In ihr fanden Valentin et al. 75 Medikationsirrtümer pro 100 Patiententage: beinahe ein „Ereignis“ pro Patient und intensivmedizinischem Behandlungstag. Knapp 1\% der untersuchten Patienten hatte einen bleibenden Schaden oder starb infolge des Medikationsirrtums [1].

Die Deutsche interdisziplinäre Vereinigung für Intensiv- und Notfallmedizin e.V. (DIVI) hat sich des Problems angenommen. Als erstes Ergebnis der eigens gegründeten Kommission Arzneimittelsicherheit wurde nun - bereits ein Jahr später - eine Empfehlung zur bundesweit einheitlichen Gestaltung von Spritzenetiketten vorgelegt.

Zwar war es schon bisher in vielen Einrichtungen der Akutmedizin (Anästhesie, Intensiv- und Notfallmedizin) gängige Praxis, in Spritzen aufgezogene Medikamente durch farbige Selbstklebeetiketten (Aufkleber) zu kennzeichnen. Allerdings existieren ein Vielzahl unterschiedlicher Systeme, teilweise sogar innerhalb eines Krankenhauses.
Der DIVI-Standard für Spritzenaufkleber ist eine Erweiterung der ISO 26825 [2]. Diese Norm der Internationalen Organisation für Standardisierung (ISO) regelt die Gestaltung von Spritzenaufklebern in der Anästhesie. Wegen der engen organisatorischen und teilweise personellen Verflechtung der akutmedizinischen Fächer in Deutschland war eine von der klinischen Anästhesie (und damit von der ISO 26825) abweichende Gestaltung in Intensiv- und Notfallmedizin einerseits und Anästhesie andererseits nicht sinnvoll [3].

In der ISO 26825 werden einzelne Medikamente bestimmten Wirkungsgruppen mit einer Kennfarbe zugeordnet (z. B. Hypnotika gelb). Zugrunde liegt die Überlegung, dass Verwechslungen innerhalb einer Wirkungsgruppe (z. B. Hypnotika) weniger schwerwiegende Folgen haben, als Verwechslungen zwischen den Gruppen (z. B. Verwechslung eines Hypnotikums mit einem Relaxans). Eine Erweiterung der ISO 26825 war notwendig, weil ansonsten zu viele der in der Intensivmedizin eingesetzten Medikamente zur Gruppe „Diverse Medikamente“ mit einheitlichem Layout (schwarze Schrift auf weißem Grund) gehört hätten.

Einzelheiten des DIVI-Standards können über die Internetpräsenz der DIVI (www.divi-org.de) eingesehen werden. Im Wesentlichen wurden für sieben weitere Medikamentengruppen die Kennfarben festgelegt und ein Basislayout für große Spritzenetiketten, die sich für 50-ml-Spritzen eignen, festgelegt.

Nun ist es an jedem, bzw. jeder einzelnen Abteilung, diese Empfehlung zur Vereinheitlichung in seinem Tätigkeitsbereich umzusetzen und damit seinerseits einen kleinen Beitrag zu noch mehr Sicherheit zu leisten. 


\section{Literatur}

1 Valentin A et al. Fehler bei der parenteralen Medikamentenverabreichung auf Intensivstationen. Deutsches Ärzteblatt 2009; 106: A771 - A777

2 International standard 26825/2009: Anaesthetic and respiratory equipment - User-applied labels for syringes containing drugs used during anaesthesia - Colours, design and performance.

www.din.de

3 Prien T. Empfehlung der DGAI zur farbigen Kennzeichnung von Spritzen. Anästh Intensivmed 2009; 50: $333-334$

\section{Korrespondenzadressen}

Prof. Dr. med. Thomas Prien

Klinik und Poliklinik für Anästhesiologie und operative Intensivmedizin

Albert-Schweitzer-Str. 33

48129 Münster

E-Mail: prien@anit.uni-muenster.de 